\title{
Down-Regulation of MiR-30c Promotes the Invasion of Non-Small Cell Lung Cancer by Targeting MTA1
}

\author{
Yang Xia ${ }^{a, c}$ Qiyou Chen ${ }^{b, c}$ Zhaopeng Zhonga Caihua Xu Chen Wua Bin Liu ${ }^{a}$ \\ Yijiang Chen ${ }^{\mathrm{a}}$ \\ aDepartment of Thoracic and Cardiovascular Surgery, The First Affiliated Hospital of Nanjing Medical \\ University, Nanjing, Jiangsu; 'Department of Thoracic Surgery, Huai'an First People's Hospital of \\ Nanjing Medical University, Huai'an, Jiangsu; 'These authors contributed equally to this work
}

\author{
Key Words \\ MicroRNA-30c • MTA1 • Invasion • NSCLC
}

\begin{abstract}
Background: The connection between microRNA expression and lung cancer development has been identified in recent literature. However, the mechanism of microRNA has been poorly elucidated in non-small-cell lung cancer (NSCLC). Methods and Results: Comparing with adjacent tissues $(n=75)$, miR-30c has a lower expression in lung cancer specimens $(n=75)$. The knockdown of miR-30c enhanced the invasion of A549 cells; meanwhile, the overexpression of miR-30c could reverse the effect of the knockdown of miR-30c in vitro. A luciferase assay revealed that miR-30c was directly bound to the $3^{\prime}$-untranslated regions (3'-UTR) of MTA1. QRT-PCR and western blot shows MTA1 was up-regulated in mRNA and protein levels. The effect taken on the invasion of NSCLC by overexpression of MTA1 works the same as downregulated miR-30c. Conclusion: miR-30c may play a pivotal role in controlling lung cancer invasion through regulating MTA1in NSCLC.
\end{abstract}

Copyright $(2013$ S. Karger AG, Basel

\section{Introduction}

Lung cancer is one of the most common causes of death among cancers, especially for male [1]. In China, lung cancer population has grown quickly over the past five years [2]. Nearly $80 \%$ were non-small cell lung cancer (NSCLC) [3, 4]. Although the methods of diagnosis and therapy have been promoted, $30-40 \%$ patients with NSCLC still have poor 
$\mathrm{Xia} /$ Chen/Zhong/Xu/Wu/Liu/Chen: Down-Regulation of MiR-30c Promotes the Invasion of NSCLC

prognosis due to the invasion of cancer cells. Therefore, elucidating the potential mechanism of metastasis would help us understand the progression and the therapy of lung cancer. Metastasis-associated protein 1 (MTA1), a key dual co-regulatory protein, belongs to the metastasis-associated protein family (including of MTA1, MTA2, and MTA3). MTA1 was regarded as an integral part of nucleosome remodeling and histone deacetylation (NuRD) complex, which was relevant to transcriptional regulation, histone deacetylation, and chromatin remodeling $[5,6]$. Lots of evidences suggested that MTA1 was up-regulated in several cancer types, consisting of carcinoma of the esophagus, breast, liver and lung [7-10], and associated with tumor progression and metastasis by interacting various cell signaling pathways.

MicroRNAs (miRNAs) were identified as an abundant class of small non-coding RNAs that played an important role in post-transcriptional regulation in various biological processes. The mature miRNAs are single-stranded RNAs including 19 to 25 nucleotides. Many functions of miRNAs related to cancers have been described in emerging literatures. For example, targeting Cyclin D1 and Ets1 by miR-9 inhibited proliferation and invasion in gastric cancer [11]. Meanwhile, Zhang et al. verified that miR-138 inhibited tumor growth through repressing expression level of EZH2 in NSCLC [12].

In our study, we mainly focused on the affection of miR-30c in NSCLC, in order to discover the potential mechanism involved in NSCLC.

\section{Material and Methods}

\section{Clinical Samples}

75 patients with NSCLC had undergone routine surgery at The First Affiliated Hospital of Nanjing Medical University from May 2012 and November 2012. NSCLC samples and the adjacent lung tissues taken from the 75 patients were collected, immediately snap frozen in liquid nitrogen, and stored at $-80{ }^{\circ} \mathrm{C}$ until RNA extraction. The tumors were classified according to World Health Organization classification. This study was approved by the Ethical Committee of The First Affiliated Hospital of Nanjing Medical University, and every patient had written informed consent.

Cell culture

A549 cell line (ATCC) was employed for the present study and was cultured in RPMI-1640 medium with $10 \%$ fetal bovine serum (Invitrogen, Carlsbad, CA) and penicillin $(100 \mathrm{U} / \mathrm{ml})$. Cells were cultured at $37^{\circ} \mathrm{C}$ with $5 \% \mathrm{CO} 2$.

\section{Isolation of total RNA and Quantitative RT-PCR}

Total RNA was extracted from collected tissues using TRIzol (Invitrogen, USA) and then both miRNA and mRNA were reversely transcribed to cDNA. The stem-loop primer for miR-30c was 5'-GTC GTA TCC AGT GCA GGG TCC GAG TAT TCG CAC TGG ATA CGA CGC TGA-3'. U6 small nuclear RNA was used for normalization. PCR reactions were performed with the following primers: for hsa-miR-30c, forward, 5'-GCC GCT GTA AAC ATC CTA CAC T-3' and reverse, 5'-GTG CAG GGT CCG AGG T-3'; and for U6, forward, 5'-CTC GCT TCG GCA GCA CA-3' and reverse, 5'-AAC GCT TCA CGA ATT TGC GT-3'. Relative expression levels of MTA1 mRNA were examined by SYBR Green real-time PCR (RT-PCR) and normalized to GAPDH. The primers for MTA1 were forward, 5'-AGC TAC GAG CAG CAC AAC GGG GT-3' and reverse, 5'-CAC GCT TGG TTT CCG AGG AT-3'; and for GAPDH, forward, 5'-CGT GGG CCG CCC TAG GCA CCA-3' and reverse, 5'-TTG GCT TAG GGT TCA GGG GGG-3'. RT-PCR was performed by using the ABI 7500 Fast Real-Time PCR system (ABI, CA, USA).

\section{Immunohistochemistry}

The tissues were fixed in $4 \%$ paraformaldehyde and cut from paraffin block to $5 \mu \mathrm{m}$ thickness. After dewaxing with xylene and rehydration with a graded series of ethanol, slides were heated in the autoclave for three minutes using citrate sodium buffer (PH 6.0) and incubated with the antibody MTA1(1:1000 dilution) at $4^{\circ} \mathrm{C}$ overnight. Blocking serum or antibody dilution buffer was used as Negative controls. The antibodies 


\section{Cellular Physiology $\quad$ Cell Physiol Biochem 2013;32:476-485

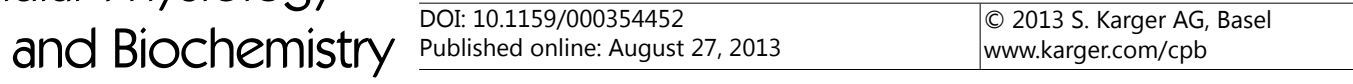 \\ $\mathrm{Xia} /$ Chen/Zhong/Xu/Wu/Liu/Chen: Down-Regulation of MiR-30c Promotes the Invasion \\ of NSCLC}

utilized before were the same as those for Western blot analysis. Photographs were taken by microcope (Nikon, ECLIPSE 50i) and software NIS-Elements v4.0. Average values of integrated optical density (IOD) were obtained from five random fields per slide by using Image-Pro Plus software (v5.0). Every data was detected three times. The number of positive staining cells showing immunoreactivity of MTA1 in ten representative microscopic fields was counted, and the percentage of positive cells was also calculated. The percentage scoring of immunoreactive tumor cells was described as follows: 0 (0 \%), 1 (1-10\%), 2 (11-50 $\%$ ), and 3 ( $>50 \%$ ). The intensity was scored as follows: 0 (negative), 1 (weak), 2 (moderate), and 3 (strong). The expression level of MTA1 was measured by multiplying the percentage and the intensity score. High expression samples means tumors with a multiplied score exceeding 4 while the others were considered to be low expression.

\section{Cell Proliferation Assay}

Cells were seeded into 96-well plates at 2000 cells/well. $20 \mu \mathrm{L}$ of 3-(4,5)-dimethylthiahiazo (-z-y1)3,5-di-phenytetrazoliumromide (MTT) $(0.5 \mathrm{mg} / \mathrm{mL})$ was added into each well. Finally, $200 \mu \mathrm{L}$ of DMSO was added to each well to dissolve the precipitate. Optical density was measured at the wavelength of $490 \mathrm{~nm}$. The data are presented as mean $\pm \mathrm{SD}$, derived from triplicate samples of at least 3 independent experiments.

Apoptosis Assay

Forty-eight hours after transfection, apoptosis in cultured cells was evaluated using annexin V labeling. An annexin V-FITC labeled Apoptosis Detection Kit (Abcam) was used according to the manufacturer's protocol.

\section{Cell Migration and invasion assay}

Cells were plated into 6-well plates and cultured with RPMI-1640 medium. After 48 hours, the cells were wounded with a pipette tip. Serum-free RPMI-1640 medium was added, and wound closure was observed for 48 hours.

Cell motility was measured using an 8- $\mu$ m-pore polycarbonate membrane Boyden chamber insert in a Transwell apparatus (Millipore, MA, USA). The transfected cells was treated with trypsin/EDTA solution, washed once with serum-containing RPMI-1640 medium. A total of $1 \times 10^{5}$ cells in $0.2 \mathrm{ml}$ serum-free RPMI1640 medium were seeded on a Transwell apparatus. RPMI-1640 containing 10\% FBS (600 $\mu$ l) was added to the lower chamber. An invasion assay was conducted following the same procedure, with the exception that the filters of the transwell chambers were coated with $45 \mu \mathrm{g}$ Matrigel (BD Biosciences; San Jose, CA, USA). Following incubation of the cells for $24 \mathrm{~h}$ at $37^{\circ} \mathrm{C}$ in a $5 \% \mathrm{CO}_{2}$ incubator, cells on the top surface of the insert were removed by wiping with a cotton swab. Cells that invaded to the bottom surface of the insert were fixed in the $100 \%$ precooling methanol for $10 \mathrm{~min}$, stained in $0.5 \%$ crystal violet for $30 \mathrm{~min}$, then rinsed in PBS and subjected to microscopic inspection. The values for invasion were obtained by counting three fields per membrane and represented the average of three independent experiments.

\section{Bioinformatics analysis}

In this study we used bioinformatics method to predict the potential targeting genes of miR-30c. As the results shown in the starBase (http://starbase.sysu.edu.cn/) database, MTA1 was the candidate gene that we picked up. The result of bioinformatic software indicated that 3'-UTR of MTA1 binds to miR-30c with the high score.

\section{Western blot}

Total proteins were prepared from the established cells, quantities using a protein assay (BCA method, Beyotime, China). Proteins were fractionated by sodium dodecyl sulfate polyacrylamide gel electrophoresis (SDS-PAGE) transferred to polyvinylidene fluoride (PVDF) membrane, blocked in 5\% dry milk at room temperature for 1 hour and immunostained with antibodies at $4^{\circ} \mathrm{C}$ overnight using anti-MTA1 (1:1000, Dizhao, Nanjing, China) and anti-GAPDH (1:5000, Kangchen, China). All results were visualized through a chemiluminescent detection system (Pierce ECL Substrate Western blot detection system, Thermo, Pittsburgh, PA) and then exposed in Molecular Imager ChemiDoc XRS System (Bio-Rad, Hercules, CA). The integrated density of the band was quantified by ImageJ software. 
Xia/Chen/Zhong/Xu/Wu/Liu/Chen: Down-Regulation of MiR-30c Promotes the Invasion

of NSCLC

Fig. 1. MiR-30c is down-regulated in NSCLC patients. The expression levels of miR-30c in human NSCLC tissues and corresponding adjacent tissues relative to U6 were determined by qRT-PCR. $(\mathrm{n}=75, \mathrm{p}<0.0067)$ Data are represented as mean \pm SD. * indicates $\mathrm{P}<0.05$.

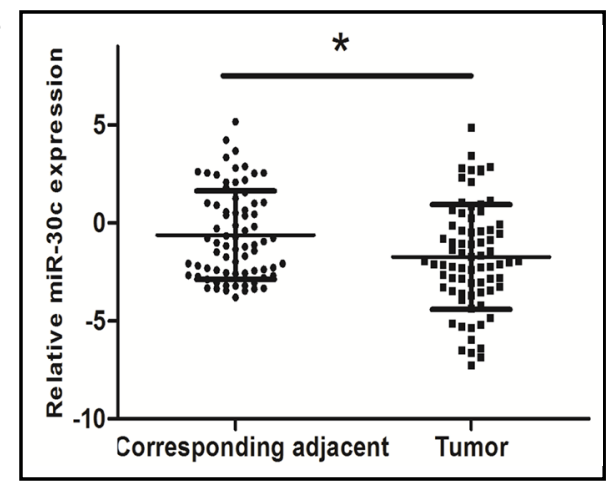

Plasmid construction and cell transduction

3'-UTR sequence of MTA1 which was predicted to interact with miR-30c or a mutant sequence with the predicted target sites was inserted into the KpnI and SacI sites of pGL3 promoter vector (Genscript, Nanjing, China). They were named pGL3-MTA1 and pGL3-MTA1-mut. The cells were plated onto 6-well plates and were transfected with $100 \mathrm{ng}$ of pGL3-MTA1 or pGL3-MTA1-mut, and miR-30c mimics (50nM) by using Lipofectamine 2000 (Invitrogen Corp, CA, USA). We normalized the differences in transfection efficiency by co-transfecting a Renilla luciferase vector pRL-SV40 (5 ng).

MTA1 gene was synthesized (purchased from Genscript, Piscataway, NJ) with restrictive digestion using Mlu I and subcloned pLV-GFP plasmid (gift from D. Beicheng Sun, University of Nanjing Medical University, China), and named pLV-GFP-MTA1. Recombinant lentivirus was generated from 293T cells using calcium phosphate precipitation. A549 cell line was transfected with lentivirus using polybrene $(8 \mathrm{ug} / \mathrm{ml})$.

Luciferase activities analysis

48 hours after transfection, luciferase actions were measured in Victor 1420 Multilabel Counter (Wallac, Finland) using Luciferase Assay System (Promega, USA) according to the manufacturer's protocol.

Statistical methods

The method of 2- $\Delta \mathrm{Ct}$ was used to analyze the results of RT-PCR in all the experiments performed in this study. Statistical analysis was performed using STATA 9.2, and presented with Graph PAD prism software. The results obtained from experiment in vitro assays are presented as mean \pm SEM from five separate experiments in triplicates per experiment, and the data was analyzed by Wilcoxon rank-sum (Mann-Whitney) test. Results were considered statistically significant at $\mathrm{P}<0.05$.

\section{Results}

MiR-30c was decreased in human lung cancer tissues

The expression of miR-30c was analyzed in both lung cancer and adjacent lung tissues by qRT-PCR. Significantly, miR-30c expression was lower in lung cancer tissues than paraneoplastic tissues (Fig. 1), which was consistent with other meta-analysis $[13,14]$. The aberrant expression level of miR-30c suggested that miR-30c might play an important role in lung cancer progression and development. Therefore, based on this expression pattern, we chose the A549 cell line to verify the effect of miR-30c.

Aberrant expression level of miR-30c affected lung cancer cell invasion in vitro

A549 cells were transfected with miR-30c mimics and anti-miR-30c respectively. The transfection efficiency was validated by qRT-PCR (Fig. 2A). Using wound healing assay, the overexpression of miR-30c could suppress A549 cell invasion, while suppressed miR-30c increases cell invasion (Fig. 2B). Matrigel invasion assay showed that overexpression of miR$30 \mathrm{c}$ attenuated A549 cell invasion, whereas the suppressed miR-30c could reverse its effect (Fig. 2C). The results suggested that miR-30c could inhibit invasion of the A549 cell line in vitro. 


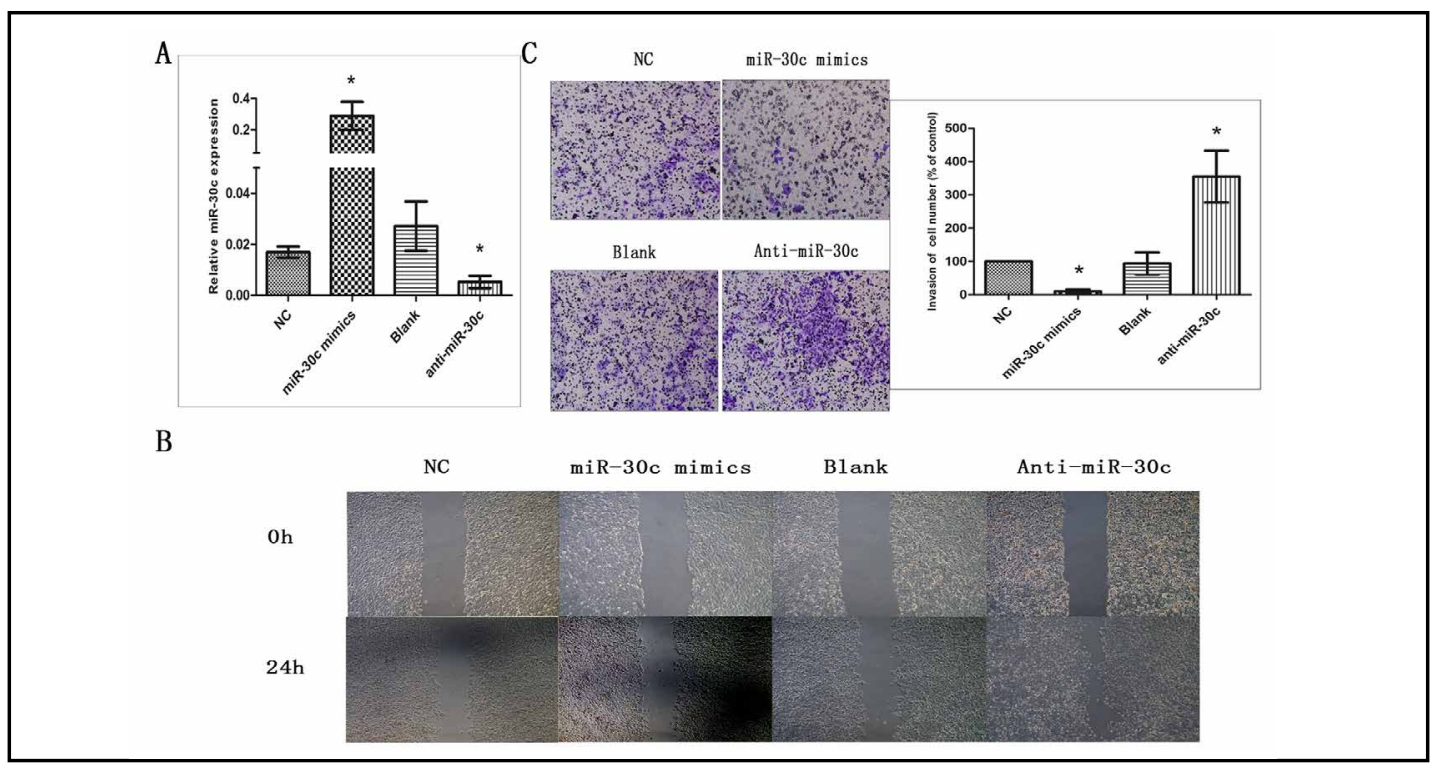

Fig. 2. Effects of miR-30c expression on A549 cells invasion. A. MiR-30c expression level in A549 cells transfected with miR-30c mimics, anti-miR-30c, control for miR-mimics (NC) and control for anti-miR (blank). The result was validated by real-time PCR. B. The images photographed at $0 \mathrm{~h}$ (upper) and $24 \mathrm{~h}$ (lower) post-wounding were shown at magnification of x200. C. Transwell assay was performed as described in Materials and Methods. Cells were treated with miR-30c mimics, anti-miR-30c, control for miR-mimics (NC) and control for anti-miR (blank) for $24 \mathrm{~h}$. The representative images of invasive cells at the bottom of the membrane stained with crystal violet were visualized as shown. The quantifications of cell migration were presented as percentage of migrated cell numbers. All experiments were performed in triplicate and presented as mean \pm SD. * indicates significant difference compared with control group $(\mathrm{P}<0.05)$. Every independent experiment was performed 3 times.

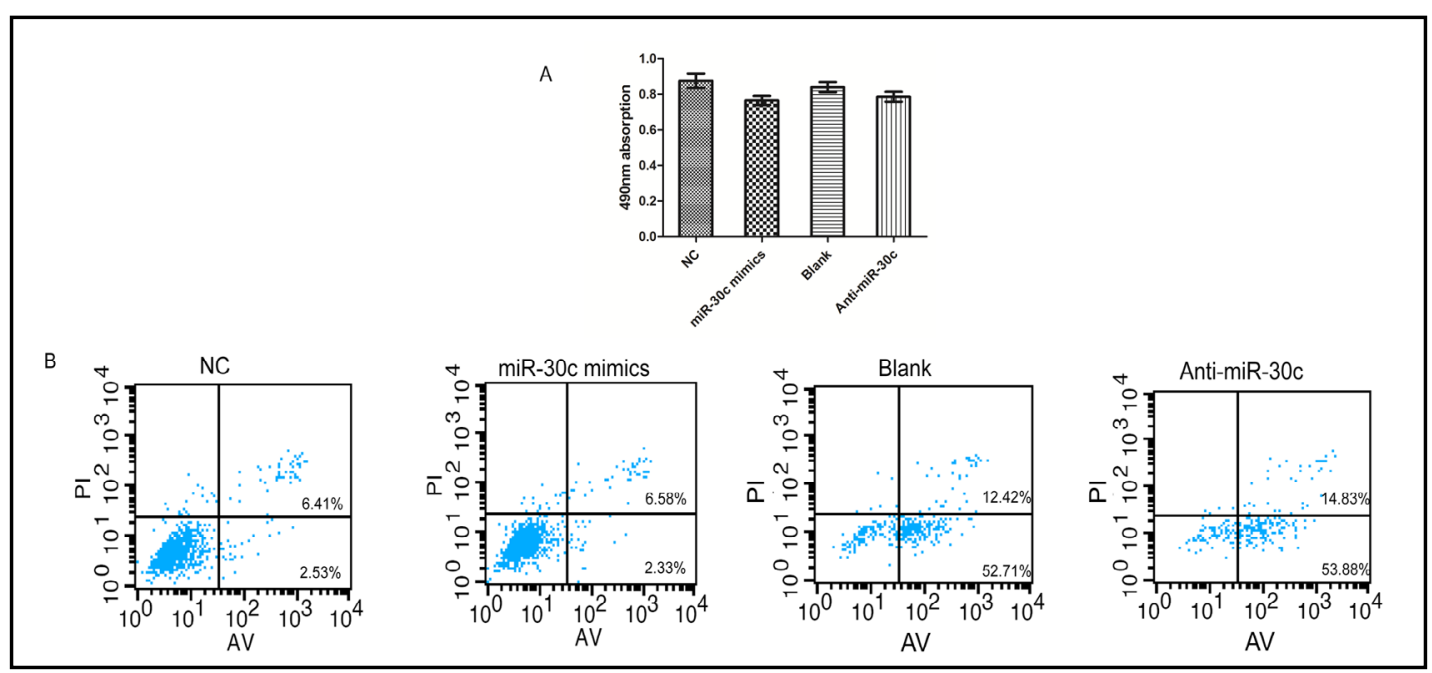

Fig. 3. MiR-30c has no affection on cell proliferation and apoptosis. A: Absorbance at $490 \mathrm{~nm}$ was presented with Mean \pm SD. B: Flow cytometry assay was performed to assess cell apoptosis. Both of the two experiment indicated no significant difference compared with the controls.

miR-30c had no affection on cell proliferation and apoptosis

The effect of miR-30c on cell proliferation was detected by using MTT assay. The assay showed that the cell survival of cells transfected with miR-30c mimics in A549 cells was no difference compared with the controls. We also used apoptosis assay to detect the effect of miR-30c on cell apoptosis. However, there was no significant difference (Fig. 3). 


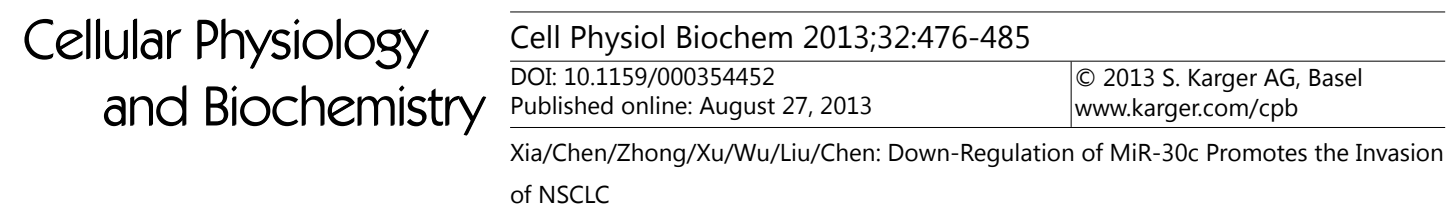

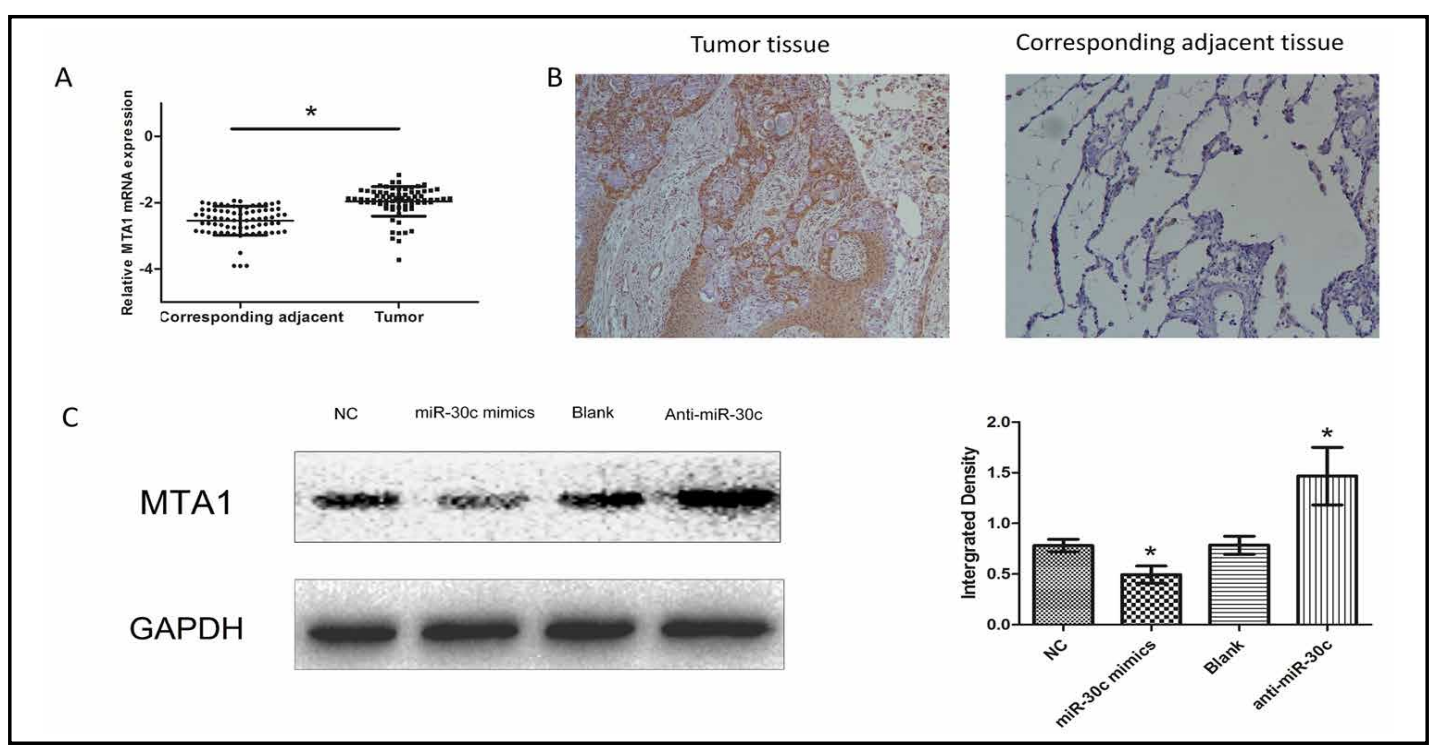

Fig. 4. Up-regulation of MTA1 in non-small cell lung cancer tissues. A, The mRNA levels of MTA1 relative to GAPDH in human NSCLC tissues and corresponding adjacent tissues were evaluated by qRT-PCR. Data were presented as scattergram of the median. * Significantly different compared with that of control $(\mathrm{P}<0.05) . \mathrm{B}, \mathrm{MTA} 1$ protein expression in tumor and corresponding adjacent tissues was detected by Immunohistochemical staining. C, MTA1 protein expression levels in A549 cells transfected with miR-30c mimics, anti-miR-30c, control for miR-mimics (NC) and control for anti-miR (blank) were analyzed by Western-blotting. GAPDH was used as a loading control. Average values of integrated optical density (IOD) were assessed by analyzing five fields per slide and recorded in the histogram. Data are represented as mean \pm SD. * indicates $\mathrm{P}<0.05$.

Table 1. Expression level of MTA1 in lung cancer and corresponding adjacent tissues. SC: squamous carcinoma; AC: adenocarcinoma; ASC: adenosquamous carcinoma; * indicates $\mathrm{P}<0.05$; ** indicates $\mathrm{P}<0.001$

\begin{tabular}{lllll}
\hline Factor & Sample N=75 & MTA1 expression & P value \\
& & Low & High & \\
\hline Gender & & & & 0.773 \\
Male & 42 & 15 & 27 & \\
Female & 33 & 11 & 22 & \\
Age(years) & & & & 0.521 \\
$<60$ & 34 & 14 & 20 & \\
$\geq 60$ & 41 & 12 & 29 & \\
Smoker & & & & 0.278 \\
No & 35 & 12 & 23 & \\
Yes & 40 & 14 & 26 & \\
Histology & & & & 0.692 \\
SC & 33 & 11 & 22 & \\
AC & 41 & 14 & 27 & \\
ASC & 1 & 1 & 0 & \\
Tumor & & & & 0.103 \\
T1/T2 & 51 & 17 & 34 & \\
T3/T4 & 24 & 14 & 10 & \\
Lymph node metastasis & & & & $0.021^{*}$ \\
N0 & 33 & 16 & 17 & \\
N1/N2/N3 & 42 & 10 & 32 & \\
Stage & & & & $0.001^{* *}$ \\
I - II & 33 & 16 & 17 & \\
III-IV & 42 & 10 & 32 & \\
\hline
\end{tabular}

miR-30c decreased MTA1 expression in NSCLC

Among hundreds of genes predicted by online miRNA target prediction algorithms, named starBase (http://starbase.sysu.edu.cn/), we focused on MTA1, which had been 
Xia/Chen/Zhong/Xu/Wu/Liu/Chen: Down-Regulation of MiR-30c Promotes the Invasion of NSCLC

Fig. 5. Effect of miR-30c on MTA1 expression by luciferase reporter assay. The potential miR-30c binding site at the 3-UTR of MTA1 mRNA was computationally predicted by starBase. A549 cells were co-transfected with miR30c mimics (or negative control) with pGL3-MTA1 (or pGL3-MTA1-mut) vector. Luciferase activity was normalized by the ratio of firefly and Renilla luciferase signals. * indicates $\mathrm{P}<0.05$.
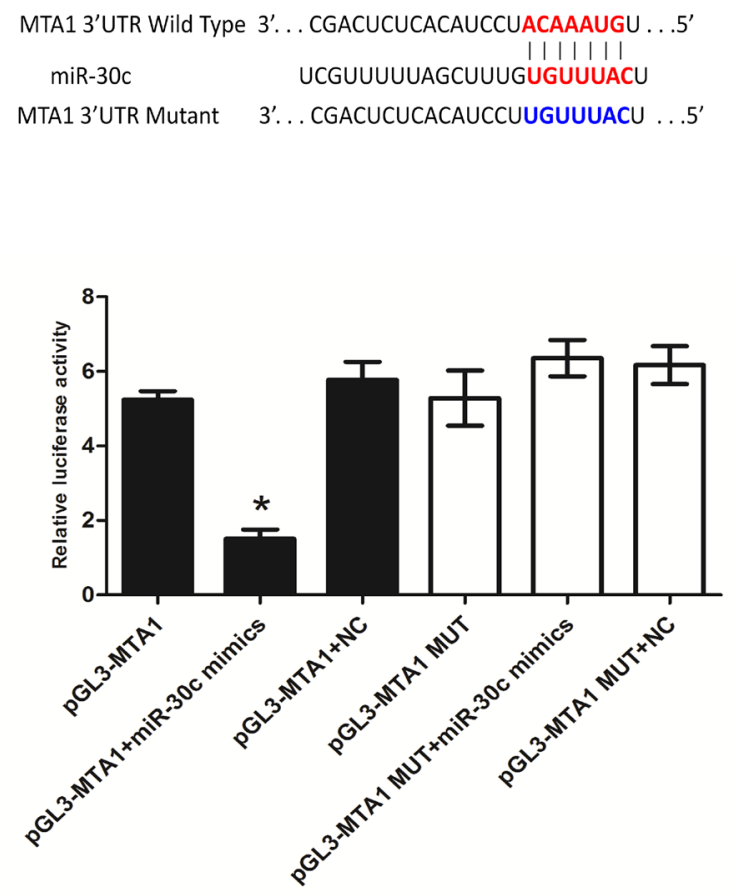

reported regulating lung cancer progression. MTA1 was up-regulated in lung cancer tissues ( $\mathrm{n}=75$, Fig. 4A and B) by using qRT-PCR and immunohistochemistry. We also discovered that MTA1 expression was significantly associated with lymph node metastasis and stage $(p<0.05$; Table 1) by using immunohistochemistry assay. We detected the expression of MTA1 responses to the changes of miR-30c expression in vitro. Western blot and qRT-PCR assays showed that over-expressed miR-30c could significantly down-regulate mRNA and protein level of MTA1, meanwhile, suppressed miR-30c had adverse effects (Fig. 4 C).

MTA1 was a direct target gene of miR-30c in A549 lung cancer cells

Despite MTA1 has been regarded as a direct target of miR-30c in this research, it was unclear whether miR-30c could directly recognize 3'-UTR of MTA1 mRNA. According to results of prediction, we cloned the 3 '-UTR fragment containing the predicted site into pGL3 luciferase reporter vector (pGL3-MTA1). The 3'-UTR fragment with mutant sequence was also cloned as a control group (pGL3-MTA1-mut) in the predicted target site. The results showed that the luciferase activity decreased in A549 cells with miR-30c mimics and pGL3MTA1 vectors. However, miR-30c mimics did not have any effect on luciferase activity when target cells were transfected with pGL3-MTA1-mut vector (Fig. 5). These data suggested that MTA1 gene was one of the direct targets of miR-30c.

Overexpression of MTA1 promoted cell invasion

Overexpression of MTA1 was confirmed in A549 by lenti-virus using western blotting (Fig. 6A).We discovered that overexpression of MTA1 significantly promoted A549 cell invasion ability (Figs. 6B and C) by using wound healing and matrigel invasion assay.

\section{Discussion}

Lots of evidences have suggested that miRNAs played an important role in carcinogenesis and cancer progression [15]. MiR-30c, regarded as a tumor inhibitor, was decreased and targeting regulaed cytoskeleton genes that involve in breast cancer cell invasion [16]. MiR-30c was also inclined in malignant peripheral nerve sheath tumors [17]. Meanwhile, 
Xia/Chen/Zhong/Xu/Wu/Liu/Chen: Down-Regulation of MiR-30c Promotes the Invasion of NSCLC

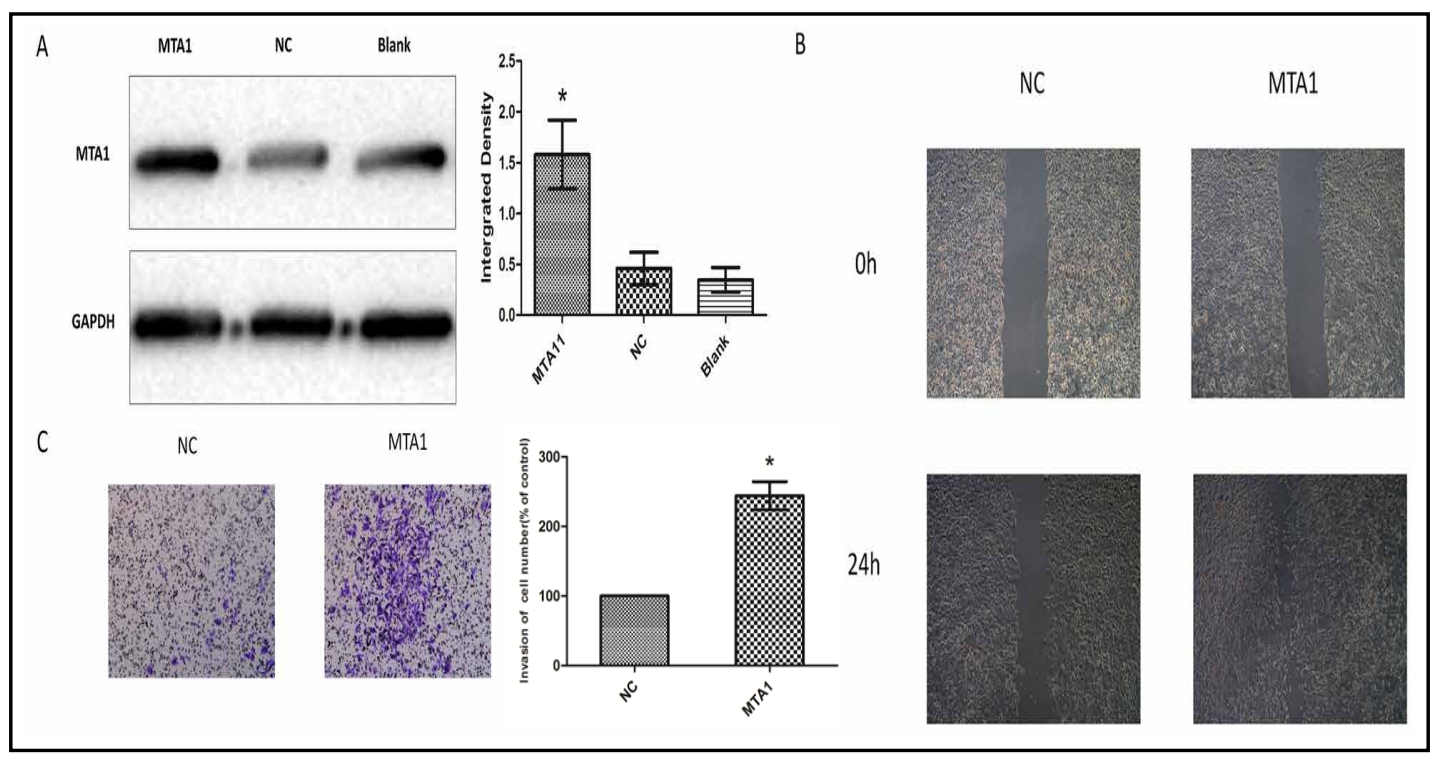

Fig. 6. Effects of over-expression of MTA1 by transfecting with lentivirus on A549 cell invasion. A, MTA1 protein expression was analyzed by Western blotting in A549 cells by transfecting with lentivirus, control lentivirus, and blank. GADPH was shown as internal control (left). Average values of integrated density were assessed by analyzing five fields per slide and recorded in the histogram (right). B, Wound healing assay of these images at $0 \mathrm{~h}$ (upper) and $24 \mathrm{~h}$ (lower) post-wounding were shown at magnification of 200x. C, Transwell assay was performed as described in Materials and Methods. MTA1 over-expressed and control lentivirus groups were respectively stained. The representative images of invasive cells at the bottom of the membrane stained with crystal violet were visualized as shown. The quantifications of cell migration were presented as percentage of migrated cell numbers. All experiments were performed in triplicate and presented as mean \pm SD. * indicates significant difference compared with control group $(\mathrm{P}<0.05)$. Every independent experiment was performed 3 times.

miR-30c inhibited expression of SERPINE 1 in human endothelial cells [18]. In human, the miR-30 family (miR-30a/b/c/d/e/f) was also reported in various cancers, such as breast [19], retinal pigment epithelial cells [20], glioma [21], and osteoblast [22]. However, recent literature indicated that aberrant expression level of miR-30c induced tumorigenesis and gefitinib resistance in lung cancer, while miR-30c was altered by EGFR and MET receptor tyrosine kinase [23]. Our study found that miR-30c negatively regulated the expression level of MTA1 in both lung cancer tissues and cell lines, suggesting that miR-30c might play an important role in the development of NSCLC. Researchers have found that the SNPs of miR30c were associated with NSCLC. In the genotype-phenotype correlation analysis, rs 928508 AG/GG genotypes were associated with a significantly decreased expression of precursor and mature miR-30c [24]. Besides, during the research in gastric cancer, Expression analysis detected that rs928508 AA showed a significantly increased level of mature miR-30c compared with GG or AG/GG genotype [25]. Thus, we considered that the SNPs of miR-30c might the mechanism which induced the down-regulation of miR-30c.

In this study, expression of miR-30c in NSCLC specimens was significantly lower than in adjacent tissues. Lower expressed miR-30c was related to the invasion ability of NSCLC, which was similar to results obtained by using the MTA1 overexpressed cell line. According to the results in luciferase reporter assay, the miR-30c was related to the invasion of NSCLC by regulating MTA1. MiRNAs can regulate the target gene expression by binding its $3^{\prime}$-UTR [2628]. However, the mechanism of miR-30c requires further research. MTA1 was an integral component of NuRD complex, and played an important role in invasive, as the same result of our study $[29,30]$. Overexpression of MTA1 was reported in carcinogenesis in human colorectal, breast, and lung cancers; it was a prognostic indicator and was associated with 


\section{Cellular Physiology $\quad$ Cell Physiol Biochem 2013;32:476-485 and Biochemistry \begin{tabular}{l|l} 
DOI: 10.1159/000354452 \\
Published online: August 27, 2013 & $\begin{array}{l}\text { (c) 2013 S. Karger AG, Basel } \\
\text { www.karger.com/cpb }\end{array}$ \\
\cline { 2 - 3 }
\end{tabular} \\ $\mathrm{Xia} /$ Chen/Zhong/Xu/Wu/Liu/Chen: Down-Regulation of MiR-30c Promotes the Invasion of NSCLC}

poor survival in esophageal, breast, and urinary cancers [31]. Recent evidence showed that miR-661 repressed MTA1 expression via binding its 3'-UTR in breast cancer [32].

Our results indicated that miR-30c was down-regulaed, while MTA1 was up-regulated in lung cancers. Ectopic miR-30c expression decreased MTA1 in mRNA and protein levels in the lung cancer cell line. We also demonstrated that miR-30c was directly bound to 3 '-UTR of MTA1. MiR-30c expression inhibited lung cancer invasion and proliferation via repression of MTA1. Furthermore, miR-30c-MTA1 pathway that we studied might be exploited in a therapeutic approach for the treatment of cancers in future.

\section{Abbreviations}

NSCLC (Non-small-cell lung cancer); 3'-UTR (3'-untranslated regions); qRT-PCR (Quantitative Real Time- Polymerase Chain Reaction); NuRD (nucleosome remodeling and histone deacetylation); FBS (fetal calf serum); EDTA (Ethylene Diamine Tetraacetic Acid).

\section{Acknowledgements}

This research was partly supported by Dr. Junwei Tang for language revision. Thanks for D. Beicheng Sun and Zhe Lin.

\section{Reference}

1 Jemal A, Siegel R, Xu J, Ward E: Cancer statistics, 2010. CA Cancer J Clin 2010;60:277-300.

-2 Yang L, Parkin DM, Ferlay J, Li L, Chen Y: Estimates of cancer incidence in china for 2000 and projections for 2005. Cancer Epidemiol Biomarkers Prev 2005;14:243-250.

3 Herbst RS, Heymach JV, Lippman SM: Lung cancer. N Engl J Med 2008;359:1367-1380.

4 Brundage MD, Davies D, Mackillop WJ: Prognostic factors in non-small cell lung cancer: A decade of progress. Chest 2002;122:1037-1057.

5 Li DQ, Pakala SB, Nair SS, Eswaran J, Kumar R: Metastasis-associated protein 1/nucleosome remodeling and histone deacetylase complex in cancer. Cancer Res 2012;72:387-394.

6 Wu M, Wang L, Li Q, Li J, Qin J, Wong J: The mta family proteins as novel histone h3 binding proteins. Cell Biosci 2013;3:1.

7 Toh Y, Kuwano H, Mori M, Nicolson GL, Sugimachi K: Overexpression of metastasis-associated mta1 mrna in invasive oesophageal carcinomas. Br J Cancer 1999;79:1723-1726.

8 Tong D, Heinze G, Schremmer M, Schuster E, Czerwenka K, Leodolter S, Zeillinger R: Expression of the human mta1 gene in breast cell lines and in breast cancer tissues. Oncol Res 2007;16:465-470.

-9 Lin C, Chen H, Wu M, Yang G, Dai J, Hu S: [expression of tumor metastasis gene mta 1 in hepatocellular carcinoma: Clinical implications]. Zhonghua wai ke za zhi [Chinese journal of surgery] 2000;38:915-917.

10 Yu Y, Wang Z, Zhang MY, Liu XY, Zhang H: Relation between prognosis and expression of metastasisassociated protein 1 in stage i non-small cell lung cancer. Interact Cardiovasc Thorac Surg 2011;12:166169.

11 Zheng L, Qi T, Yang D, Qi M, Li D, Xiang X, Huang K, Tong Q: Microrna-9 suppresses the proliferation, invasion and metastasis of gastric cancer cells through targeting cyclin $\mathrm{d} 1$ and ets1. PloS one 2013;8:e55719.

12 Zhang H, Zhao M, Lv Z, Zhang X, Qin X, Wang H, Wang S, Su J, Lv X, Liu H, Du W, Zhou W, Chen X, Fei K: Mir138 inhibits tumor growth through repression of ezh2 in non-small cell lung cancer. Cell Physiol Biochem 2013;31:56-65.

13 Vosa U, Vooder T, Kolde R, Vilo J, Metspalu A, Annilo T: Meta-analysis of microRNA expression in lung cancer. Int J Cancer 2013;132:2884-2893. 


\section{Cellular Physiology Cell Physiol Biochem 2013;32:476-485

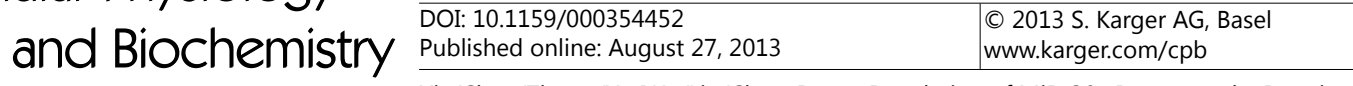 \\ $\mathrm{Xia} /$ Chen/Zhong/Xu/Wu/Liu/Chen: Down-Regulation of MiR-30c Promotes the Invasion of NSCLC}

14 Guan P, Yin Z, Li X, Wu W, Zhou B: Meta-analysis of human lung cancer microrna expression profiling studies comparing cancer tissues with normal tissues. J Exp Clin Cancer Res 2012;31:54.

- 15 Lu J, Getz G, Miska EA, Alvarez-Saavedra E, Lamb J, Peck D, Sweet-Cordero A, Ebert BL, Mak RH, Ferrando AA, Downing JR, Jacks T, Horvitz HR, Golub TR: Microrna expression profiles classify human cancers. Nature 2005;435:834-838.

-16 Bockhorn J, Yee K, Chang YF, Prat A, Huo D, Nwachukwu C, Dalton R, Huang S, Swanson KE, Perou CM, Olopade OI, Clarke MF, Greene GL, Liu H: Microrna-30c targets cytoskeleton genes involved in breast cancer cell invasion. Breast Cancer Res Treat 2013;137:373-382.

-17 Presneau N, Eskandarpour M, Shemais T, Henderson S, Halai D, Tirabosco R, Flanagan AM: Microrna profiling of peripheral nerve sheath tumours identifies mir-29c as a tumour suppressor gene involved in tumour progression. Br J Cancer 2013;108:964-972.

18 Marchand A, Proust C, Morange PE, Lompre AM, Tregouet DA: Mir-421 and mir-30c inhibit serpine 1 gene expression in human endothelial cells. PloS one 2012;7:e44532.

19 Ouzounova M, Vuong T, Ancey PB, Ferrand M, Durand G, Le-Calvez Kelm F, Croce C, Matar C, Herceg Z, Hernandez-Vargas H: Microrna mir-30 family regulates non-attachment growth of breast cancer cells. BMC genomics 2013;14:139.

20 Haque R, Chun E, Howell JC, Sengupta T, Chen D, Kim H: Microrna-30b-mediated regulation of catalase expression in human arpe-19 cells. PloS one 2012;7:e42542.

21 Quintavalle C, Donnarumma E, Iaboni M, Roscigno G, Garofalo M, Romano G, Fiore D, De Marinis P, Croce CM, Condorelli G: Effect of mir-21 and mir-30b/c on trail-induced apoptosis in glioma cells. Oncogene 2012, Epub ahead of print.

22 Wu T, Zhou H, Hong Y, Li J, Jiang X, Huang H: Mir-30 family members negatively regulate osteoblast differentiation. J Biol Chem 2012;287:7503-7511.

-23 Garofalo M, Romano G, Di Leva G, Nuovo G, Jeon YJ, Ngankeu A, Sun J, Lovat F, Alder H, Condorelli G, Engelman JA, Ono M, Rho JK, Cascione L, Volinia S, Nephew KP, Croce CM: Egfr and met receptor tyrosine kinase-altered microrna expression induces tumorigenesis and gefitinib resistance in lung cancers. Nat Med 2012;18:74-82.

24 Hu Z, Shu Y, Chen Y, Chen J, Dong J, Liu Y, Pan S, Xu L, Xu J, Wang Y, Dai J, Ma H, Jin G, Shen H: Genetic polymorphisms in the precursor microrna flanking region and non-small cell lung cancer survival. Am J Respir Crit Care Med 2011;183:641-648.

25 Mu YP, Su XL: Polymorphism in pre-mir-30c contributes to gastric cancer risk in a chinese population. Med Oncol 2012;29:1723-1732.

26 Qin W, Ren Q Liu T, Huang Y, Wang J: MicroRNA-155 is a novel suppressor of ovarian cancer-initiating cells that targets CLDN1. FEBS lett 2013;587:1434-1439.

27 Miao LJ, Huang SF, Sun ZT, Gao ZY, Zhang RX, Liu Y, Wang J: Mir-449c targets c-myc and inhibits NSCLC cell progression. FEBS lett 2013 May 2;587:1359-1365.

28 Li J, Wang Y, Luo J, Fu Z, Ying J, Yu Y, Yu W: Mir-134 inhibits epithelial to mesenchymal transition by targeting foxm1 in non-small cell lung cancer cells. FEBS lett 2012;586:3761-3765.

29 Li S, Tian H, Yue W, Li L, Gao C, Si L, Li W, Hu W, Qi L, Lu M: Down-regulation of mta1 protein leads to the inhibition of migration, invasion, and angiogenesis of non-small-cell lung cancer cell line. Acta Biochim Biophys Sin 2013;45:115-122.

-30 Wang H, Fan L, Wei J, Weng Y, Zhou L, Shi Y, Zhou W, Ma D, Wang C: Akt mediates metastasis-associated gene 1 (mta1) regulating the expression of e-cadherin and promoting the invasiveness of prostate cancer cells. PloS one 2012;7:e46888.

- 31 Cheng CW, Liu YF, Yu JC, Wang HW, Ding SL, Hsiung CN, Hsu HM, Shieh JC, Wu PE, Shen CY: Prognostic significance of cyclin d1, beta-catenin, and mta1 in patients with invasive ductal carcinoma of the breast. Ann Surg Oncol 2012;19:4129-4139.

-32 Reddy SD, Pakala SB, Ohshiro K, Rayala SK, Kumar R: Microrna-661, a c/ebpalpha target, inhibits metastatic tumor antigen 1 and regulates its functions. Cancer Res 2009;69:5639-5642. 
After the publication of the manuscript by Xia et al., entitled 'Down-regulation of MiR-30c promotes the Invasion of Non-Small Cell Lung Cancer by Targeting MTA1' [Cell Physiol Biochem 2013 Aug 27;32(2):476-485. (DOI: 10.1159/000354452)], we were informed about a minor mistake in published Table 1.

Despite the error, the main text and legends are all correct. The p value of the existing Table 1 has been replaced. Please, accept our apologies and refer to the correct corresponding Table 1 that we provide in this Erratum. Legends are the same as in the Original article.

Table1. Expression level of MTA1 in lung cancer and corresponding adjacent tissues

\begin{tabular}{|c|c|c|c|c|}
\hline \multirow{3}{*}{$\begin{array}{l}\text { Factor } \\
\text { Gender }\end{array}$} & \multirow[t]{3}{*}{ Sample N=75 } & \multicolumn{2}{|c|}{ MTA1 expression } & \multirow{3}{*}{$\begin{array}{c}\text { P value } \\
0.830\end{array}$} \\
\hline & & Low & High & \\
\hline & & & & \\
\hline Male & 42 & 15 & 27 & \\
\hline Female & 33 & 11 & 22 & \\
\hline Age(years) & & & & 0.281 \\
\hline$<60$ & 34 & 14 & 20 & \\
\hline$\geq 60$ & 41 & 12 & 29 & \\
\hline Smoker & & & & 0.948 \\
\hline No & 35 & 12 & 23 & \\
\hline Yes & 40 & 14 & 26 & \\
\hline Histology & & & & 0.384 \\
\hline SC & 33 & 11 & 22 & \\
\hline $\mathrm{AC}$ & 41 & 14 & 27 & \\
\hline ASC & 1 & 1 & 0 & \\
\hline Tumor & & & & 0.724 \\
\hline $\mathrm{T} 1 / \mathrm{T} 2$ & 51 & 17 & 34 & \\
\hline $\mathrm{T} 3 / \mathrm{T} 4$ & 24 & 9 & 15 & \\
\hline Lymph node metastasis & & & & $0.026^{*}$ \\
\hline N0 & 33 & 16 & 17 & \\
\hline N1/N2/N3 & 42 & 10 & 32 & \\
\hline Stage & & & & $0.026^{*}$ \\
\hline $\mathrm{I}-\Pi$ & 33 & 16 & 17 & \\
\hline III-IV & 42 & 10 & 32 & \\
\hline $\begin{array}{l}\text { SC: squamous carcinoma } \\
* \text { indicates } \mathrm{P}<0.05\end{array}$ & adenocarcil & ASC: & lamo & cinoma; \\
\hline
\end{tabular}

